\title{
Heavy-Atom Tunneling in the Covalent/Dative Bond Complexation of Cyclo[18]carbon-piperidine
}

\author{
Ashim Nandi* and Jan M. L.Martin* \\ Department of Molecular Chemistry and Materials Science, Weizmann Institute of Science, \\ 7610001 Reḥovot, Israel.Email: ashim.nandi@weizmann.ac.il, gershom@weizmann.ac.il
}

KEYWORDS: Quantum Mechanical Tunneling; Kinetic Isotope Effect; Cyclo[18]carbon; Dative Bond; Noncovalent Interactions.

ABSTRACT. Recent quantum chemical computations demonstrated the electron-acceptance behavior of this highly reactive cyclo[18]carbon $\left(\mathrm{C}_{18}\right)$ ring with piperidine (pip). The $\mathrm{C}_{18}$-pip complexation exhibited a double-well potential along the $\mathrm{N}-\mathrm{C}$ reaction coordinate, forming a van der Waals (vdW) adduct and a more stable, strong covalent/dative bond (DB) complex by overcoming a low activation barrier. By means of direct dynamical computations using canonical variational transition state theory (CVT), including the small-curvature tunneling (SCT), we show the conspicuous role of heavy atom quantum mechanical tunneling (QMT) in the transformation of vdW to DB complex in the solvent phase near absolute zero. Below $50 \mathrm{~K}$, the reaction is entirely driven by QMT, while at $30 \mathrm{~K}$, the QMT rate is too rapid $\left(k_{\mathrm{T}} \sim 0.02 \mathrm{~s}^{-1}\right)$, corresponding to a halflife time of $38 \mathrm{~s}$, indicating that the vdW adduct will have a fleeting existence. We also explored 
the QMT rates of other cyclo[n]carbon-pip systems. This study sheds light on the decisive role of QMT in the covalent/DB formation of the $\mathrm{C}_{18}$-pip complex at cryogenic temperatures.

\section{Introduction}

Due to its unique electronic and structural features, the newly synthesized $s p$-hybridized cyclo[18]carbon $\left(\mathrm{C}_{18}\right)$ ring has sparked widespread attention to both theoreticians and experimentalists since its first experimental observation in condensed media in $2019 .{ }^{1}$ The successful in-situ generation and characterization of this decades-old elusive $\mathrm{C}_{18}$ ring via atom manipulation by an atomic force microscope (AFM) tip was a landmark study because of the potential to be an alternate candidate for pure carbon allotropes. ${ }^{2-4}$ Following this experimental feat, several theoretical studies have explored the geometrical structure and stability of the $\mathrm{C}_{18}$ ring in the gas phase $\mathrm{e}^{5-10}$ and more importantly, a series of interesting properties have been highlighted, such as the electronic and transport properties, ${ }^{8,11-15}$ double aromatic character, ${ }^{16-18}$ dynamics behavior $^{6}$ and so on - most of these studies revolved around the noncovalent interaction of $\mathrm{C}_{18}$ with other elements or molecular entities.

In a recent combined experimental and theoretical study by Hobza's group, ${ }^{19}$ it was shown that an $\mathrm{sp}^{2}$-hybridized carbon allotrope, namely $\mathrm{C}_{60}$, forms a strong dative $\mathrm{N} \rightarrow \mathrm{C}$ dative bond with piperidine (pip), thereby explaining the long-standing question on the enhanced stability of $\mathrm{C}_{60}$ with pip compared to other organic or inorganic solvents. ${ }^{20,21}$ In contrast, planar carbon allotropes (graphene and nanotubes) forms only noncovalent interaction with pip. ${ }^{22}$ Stimulated by these works, the same group theoretically studied the electron-acceptance potential of $\mathrm{C}_{18}$ with pip in the gas phase and continuum solvent (using the pip dielectric constant). ${ }^{23}$ According to their DFT computations at the $\omega \mathrm{B} 97 \mathrm{XD} / \mathrm{def} 2-\mathrm{TZVPP}$ level, the $\mathrm{C}_{18}$-pip complex was predicted to exhibit 
double-well potentials along the $\mathrm{N}-\mathrm{C}$ reaction coordinate, first forming a weak van der Waals (vdW) adduct with a long N-C bond (3.006 $\AA$ ), followed by a thermodynamically stable strong dative bond (DB) complex with a short N-C bond (1.501 $\AA$ ). The vdW $\rightarrow$ DB transformation were low (activation barrier $\Delta E^{\ddagger}=3.2 \mathrm{kcal} \mathrm{mol}^{-1}$ ) and highly exergonic (reaction energy $\Delta E_{\mathrm{r}}=-12.6$ $\mathrm{kcal} \mathrm{mol}^{-1}$ ) in the gas phase, taking vdW complex as a reference. Extra stabilization of the $\mathrm{C}_{18}$-pip complex was predicted upon moving to the solvent phase. NBO analysis and molecular dynamics simulation further revealed the stability of the DB complex at room temperature.

Additionally, they have investigated the stability of other hypothetical cyclo[n]carbon systems with pip. Noteworthy, prior to the vdW complexation, their computations also predicted a stable hydrogen-bonded complex where hydrogen from $\mathrm{N}-\mathrm{H}$ forms a bond with $\mathrm{C}_{18}$ and its relative energy lies $1 \mathrm{kcal} \mathrm{mol}^{-1}$ above the vdW complex in the gas phase. Encouraged by the low activation barrier for the $\mathrm{vdW} \rightarrow \mathrm{DB}$ process in $\mathrm{C}_{18}$-pip and more importantly by the high exergonicity of the reaction, which may yield a narrow effective barrier width in accord with Hammond's postulate, ${ }^{24}$ we wondered if this reaction can be driven by heavy-atom quantum mechanical tunneling (QMT) even close to absolute zero.

Reactions involving QMT by heavy atoms (mostly second-row elements of the periodic table) ${ }^{25-}$ ${ }^{28}$ are relatively rare compared to light hydrogen tunneling-based reactions; however, recent years have witnessed the slow emergence of heavy-atom QMT, especially in organic chemistry..$^{25,28,29}$ This nonclassical QMT phenomenon is a well-known effect that significantly accelerates reaction rates by passing through the potential barrier, instead of crossing over it. ${ }^{30,31}$ The extent to which QMT occurs in a chemical reaction can be approximated according to $P \sim e^{-w \sqrt{\Delta E^{\ddagger} m} / \hbar}$, where $P$ is the tunneling probability, $w$ the barrier width, $\Delta E^{\ddagger}$ is the activation energy, and $m$ is the mass of the moving parts of the molecule. ${ }^{26,27}$ Clearly, among these three factors that determine the 
tunneling probability, the barrier width has the most decisive influence on the tunneling probability. Indeed, several experimental and/or computational studies have demonstrated that the characteristic features of reactions driven by heavy-atom QMT are their low and narrow barriers..$^{25,28,29}$ The few documented cases include pericyclic ${ }^{32}$ and degenerate rearrangement reactions involving carbon, ${ }^{33-35}$ fluoride $^{36,37}$ and boron ${ }^{38}$ tunneling. Carbon and nitrogen tunneling in highly exergonic reactions in reactive intermediate species, such as carbene or nitrene, has also been reported. ${ }^{39-41}$ Recently, boron atom tunneling has been predicted in the highly exergonic reaction involving the $\mathrm{N}-\mathrm{B}$ bond-stretch isomerization of nitrile-boron halides, whereby the metastable vdW adduct isomerizes to a global minimum covalent/dative bond complex. ${ }^{42}$

In this work, through direct dynamics computations, we show the dominant role of heavy-atom tunneling in the transformation of $\mathrm{vdW} \rightarrow \mathrm{DB}$ in the $\mathrm{C}_{18}$-pip complex near absolute zero in the solvent phase. We also extend our tunneling results to other cyclo[n]carbon-pip $(n=14,16,18$, 20 and 22). This study may elucidate the possibility of leveraging the role of QMT in the dative/covalent functionalization of the $\mathrm{C}_{18}$-pip complex.

\section{Computational Methods}

All of the DFT electronic structure calculations were performed at the M06-2X ${ }^{43} / \mathrm{def} 2-\mathrm{TZVP}$ level with the Gaussian 16 program suite. ${ }^{44}$ This level of theory was chosen since it correctly reproduces the observed polyynic structure of cyclo[18] carbon..$^{6,8}$ In addition, it was shown to closely match the minimum potential energy surface (PES) of $\mathrm{C}_{18}$ against DLPNO-CCSD(T)-F12 computations, ${ }^{6}$ which is crucial for our accurate direct dynamical studies. The SCF convergence criteria were set using the keyword "opt = tight", which sets the maximum and root-mean-square (RMS) forces to $1.5 \times 10^{-5}$ and $1.0 \times 10^{-5}$ hartree/bohr, and the maximum and RMS displacements 
to $6.0 \times 10^{-5}$ and $4.0 \times 10^{-5}$ bohr, respectively. The "ultrafine" grid, which is a pruned direct product of a 99 points Euler-MacLaurin radial grid combined with a 590-point Lebedev angular grid, ${ }^{45}$ was employed for all DFT calculations. Intrinsic reaction coordinate (IRC) calculations were conducted to confirm that the transition state is smoothly connected to the reactant and product side.

The semiclassical rate constants were computed using canonical variational transition state theory (CVT), ${ }^{46}$ while the tunneling rates were accounted using the highly demanding multidimensional small curvature tunneling (SCT) method..$^{47,48}$ We refer to the semiclassical CVT and the tunneling-corrected SCT rates as $k_{\mathrm{SC}}$ and $k_{\mathrm{T}}$. Polyrate $17^{49}$ was used to compute all the rate constants, with Gaussrate $17^{50}$ serving as the interface between Polyrate and Gaussian. The PageMcIver algorithm ${ }^{51}$ with a gradient and a Hessian step size of 0.002 and 0.018 Bohr (the smallest standard recommended step size for Polyrate calculation) ${ }^{49}$ was employed to map the reaction energy path for all the studied reactions. Quantized reactant state tunneling (QRST) ${ }^{52}$ calculations were used to obtain accurate rate constants at the sub-cryogenic temperatures. Geometry optimization and the rate constant calculations in the solvent phase were taken into account using the integral equation-formalism polarizable continuum model (IEFPCM) ${ }^{53}$ with a dielectric constant of 5.9 (a value for piperidine solvent). For the sake of reproducibility of our QMT computations, and due to the number of keywords involved that can slightly affect the rates, a sample Polyrate input file is provided in the Supporting Information.

All of the energetics, namely binding (BE), activation $\left(\Delta E^{\ddagger}\right)$, and the reaction $\left(\Delta E_{\mathrm{r}}\right)$ energies reported throughout this work are in the continuum piperidine solvent phase mentioned above unless otherwise mentioned and include the zero-point energy correction. 


\section{Results and Discussion}

As a starting point, we considered the double-well potential of the donor-acceptor $\mathrm{C}_{18}$-pip complex reported by Hobza and co-workers described above. Our gas-phase computations at the M06-2X/def2-TZVP level yield an N-C long-bond and short-bond complex with a bond distance of $3.016 \AA$ and $1.496 \AA$, and the corresponding binding energies (BE, without zero-point energy correction) are -3.0 and $-12.0 \mathrm{kcal} \mathrm{mol}^{-1}$, characteristics of a van der Waals (vdW) complex and a covalent/dative bond (DB) for the former and latter. These two distinct minima are separated by a low threshold barrier $\left(\Delta E^{\ddagger}\right)$ of $3.5 \mathrm{kcal} \mathrm{mol}^{-1}$ from the vdW complex, with a corresponding reaction energy $\left(\Delta E_{\mathrm{r}}\right)$ of $-9.1 \mathrm{kcal} \mathrm{mol}^{-1}$, in close agreement with the reported DFT and DLPNO-CCSD(T) computations, ${ }^{23}$ indicating the suitability of our selected level of theory. As mentioned above, inclusion of implicit solvent was shown to stabilize the $\mathrm{C}_{18}$-pip and indeed, upon transitioning our computations to the solvent phase, the threshold barrier $\left(2.2 \mathrm{kcal} \mathrm{mol}^{-1}\right)$ is significantly lowered and the reaction energy $\left(-17.7 \mathrm{kcal} \mathrm{mol}^{-1}\right)$ becomes more exergonic, indicating the extra stability of the transition state and the DB complex exerted by the solvent as compared to vdW adduct. Figure 1 depicts the $\mathrm{N}-\mathrm{C}$ bond distances and the energetics of the double-potential profile of $\mathrm{C}_{18}-$ pip in the pip solvent. 


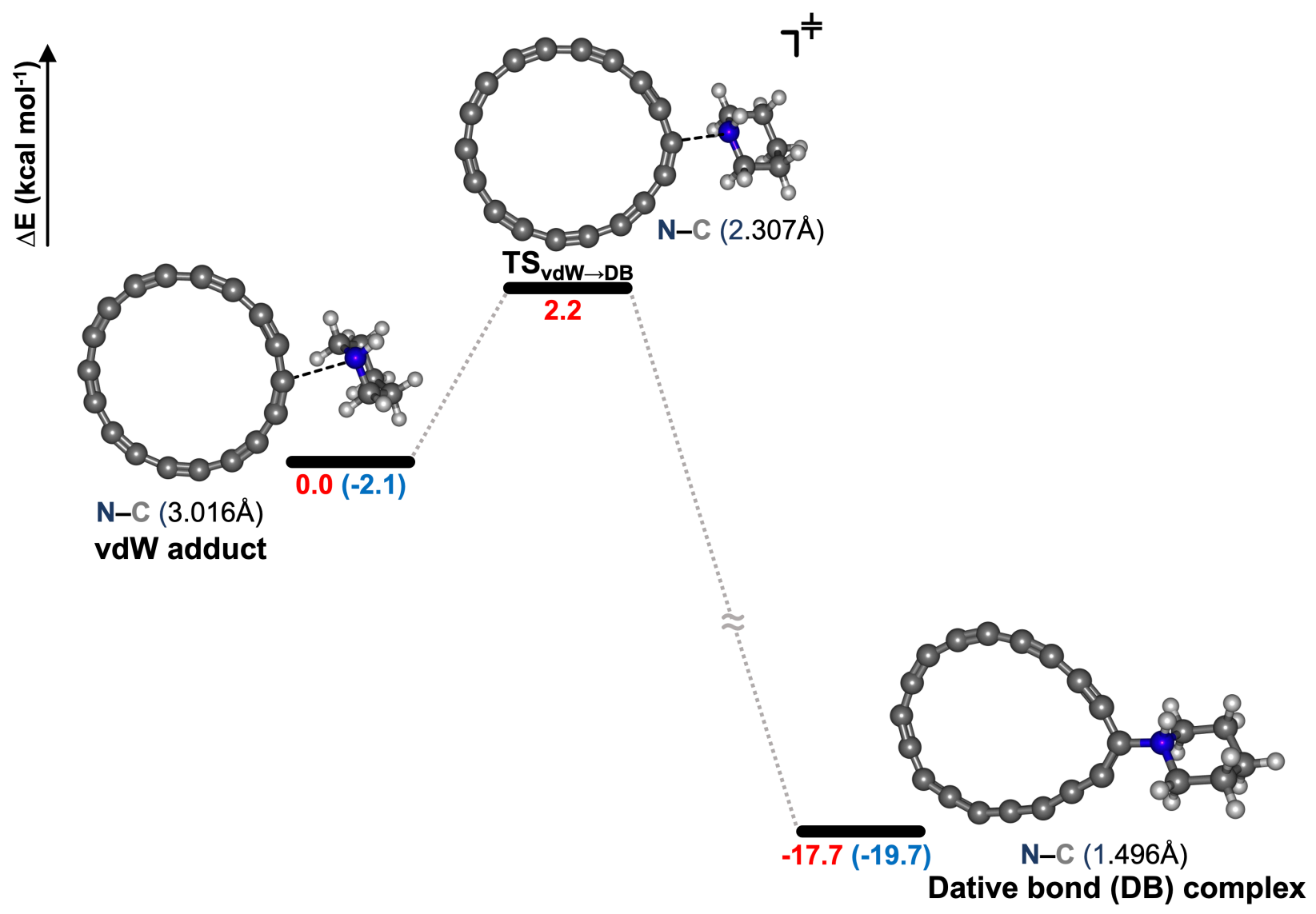

Figure 1. Double-well potential of $\mathrm{C}_{18}$-pip complex in the piperidine solvent along with $\mathrm{N}-\mathrm{C}$ bond distances. The relative energies are indicated in red and the binding energies within parenthesis in blue.

Considering the low threshold barrier and high exergonicity of the above reaction, we embarked on investigating accurate QMT rates of this chemical transformation. Our computed tunneling rate constants reveal that the reaction is wholly dominated by ground-state QMT near absolute zero. In fact, at $4 \mathrm{~K}$, the calculation shows that the classical over-the-barrier process is impossible, with a $k_{\mathrm{SC}}$ of $\sim 7 \times 10^{-110} \mathrm{~s}^{-1}$. However, the assistance of QMT speeds up the reaction with a $k_{\mathrm{T}}$ of $\sim 9 \times 10^{-}$ ${ }^{6} \mathrm{~S}^{-1}$, which should lead to an experimentally observable rate. It can also be seen from Table 1 that as the temperature increases toward the $30 \mathrm{~K}$ sublimation temperature of the argon matrix, the 
tunneling rate increases rapidly, yielding a $k_{\mathrm{T}}$ of $0.02 \mathrm{~s}^{-1}$, corresponding to a half-life $t_{1 / 2}$ (calculated as $\left.t_{1 / 2}=\ln 2 / k\right)$ of $\sim 38 \mathrm{~s}$. This means that if we can observe the transformation of $\mathrm{vdW} \rightarrow \mathrm{DB}$ in an argon matrix doped with pip solvent, the vdW adduct will have a fleeting existence and only the DB complex will be detected and isolated under matrix isolation spectroscopy. The transient behavior of the vdW complex can likewise be attributed to a similar case of quantum tunneling instability. ${ }^{54}$ Noteworthy, our gas-phase reaction rates also reveal that QMT completely drives this reaction at low temperatures (See Supporting Information for rates table) as well as that, at the liquid $\mathrm{N}_{2}$ temperature $(77 \mathrm{~K})$, the vdW adduct $\left(t_{1 / 2}=0.6 \mathrm{~s}\right)$ will have a fleeting existence. However, its $k_{\mathrm{T}}$ rates are slower compared to those in solvent, as expected due to the higher barrier and low exergonicity of the reaction (vide supra).

Table 1. Semiclassical $\left(k_{\mathrm{SC}}\right)$ and tunneling-corrected $\left(k_{\mathrm{T}}\right)$ rate constants in $\mathrm{s}^{-1}$, and half-lives $\left(t_{1 / 2}\right)$ in seconds for $\mathrm{LB}$ to $\mathrm{SB}$ transformation in $\mathrm{C}_{18}$-pip adducts from 4 to $30 \mathrm{~K}$.

\begin{tabular}{llll}
\hline $\mathrm{T}$ & $k_{\mathrm{SC}}$ & $k_{\mathrm{T}}$ & $t_{1 / 2}$ \\
\hline 4 & $7 \times 10^{-110}$ & $9 \times 10^{-6}$ & $8 \times 10^{4}$ \\
6 & $10^{-69}$ & $9 \times 10^{-6}$ & $8 \times 10^{4}$ \\
8 & $10^{-49}$ & $10^{-5}$ & $7 \times 10^{4}$ \\
10 & $10^{-37}$ & $2 \times 10^{-5}$ & $5 \times 10^{4}$ \\
20 & $2 \times 10^{-13}$ & $4 \times 10^{-4}$ & $4 \times 10^{4}$ \\
30 & $2 \times 10^{-5}$ & 0.02 & 38 \\
\hline
\end{tabular}

The Arrhenius plot depicted in Figure 2 clearly shows a divergence of the tunneling-corrected $k_{\mathrm{T}}$ rates (curved line) from the semiclassical $k_{\mathrm{SC}}$ rate constants (straight line) and reaching a plateau as the temperature is lowered, further strengthening the case for QMT in the studied system. 


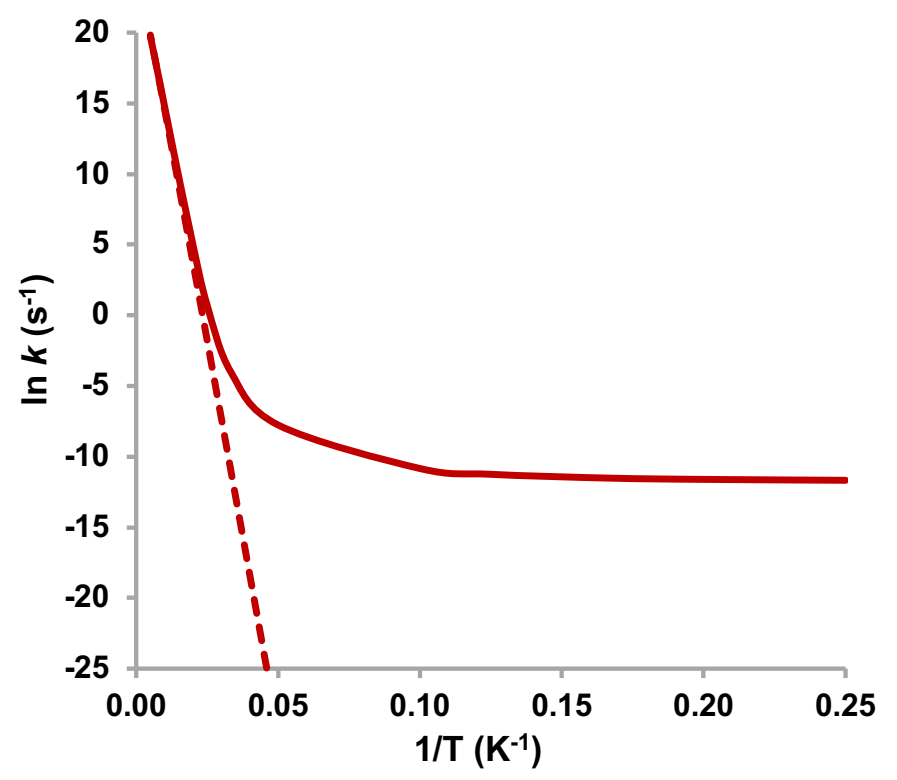

Figure 2. Arrhenius plot of the $k_{\mathrm{SC}}$ (dash) or $k_{\mathrm{T}}$ (solid line) against the inverse of temperature for a temperature range of 4 to $200 \mathrm{~K}$.

Up to about $50 \mathrm{~K}$, the role of QMT still dominates the overall reaction rate, but in this regime, excited vibrational levels of the molecules acquire significant population and QMT occurs from there by a thermally activated tunneling process. ${ }^{55}$ Note that, as opposed to hydrogen tunnelingbased reactions where the vibrational energy levels are well-separated, the energy levels in case of heavy-atom tunneling are compact and closely spaced, making the molecules easily accessible to higher vibrational energy levels even with a small amount of heat; therefore, we see a softer concave Arrhenius graph in the thermally-activated tunneling regime.

We further analyzed the kinetic isotope effect (KIE), a standard probe for the 'fingerprint' of QMT in a chemical reaction. Our computed tunneling corrected KIEs of the atoms involved along the reaction axis, i.e., nitrogen and carbon of the $\mathrm{N}-\mathrm{C}$ bond (KIE calculated as ${ }^{12} \mathrm{C} /{ }^{13} \mathrm{C}$ for carbon and ${ }^{14} \mathrm{~N} /{ }^{15} \mathrm{~N}$ for nitrogen) gives a maximum KIE of 1.41 and 2.99 at $4 \mathrm{~K}$, respectively, indicating a clear case of heavy-atom QMT; however, among them the exceptionally higher KIE value of 
nitrogen (possibly a record for this atom) suggest that it is the "tunneling-determining atom"56 i.e., the atom with the most dominant influence on the tunneling rates. As the reaction involves significant deformation of the $\mathrm{C}_{18}$ ring during the formation of the DB complex, we also computed the KIE on the ring carbons by substituting all of the 18 carbons by their heavier isotope in order to maintain the symmetry during the reaction progress. Taken like this, it produces the most KIE, a surprisingly big 2.58 at $4 \mathrm{~K}$, reflecting that all the carbons participate in the tunneling process. Furthermore, we also computed the KIE of hydrogen (H/D) attached to the nitrogen, which exhibits a large displacement during the reaction progress, and obtained a value of 2.21 at $4 \mathrm{~K}$, giving an indication of hydrogen tunneling. However, due to its small atomic mass and the large mass disparity with its isotopologues (H/D), the KIE cannot be compared to the ratio of heavier atom counterparts; but it is clear that within the heavy atoms, the nitrogen of the pip has a more decisive influence on the overall QMT rate.

We have also explored the double-well potentials of the complexes formed by analogs of $\mathrm{C}_{18}$, namely, $\mathrm{C}_{14}$ to $\mathrm{C}_{22}$ (see Table 2). All these systems considered here are closed-shell singlet ground states..$^{23,57,58}$ The $\mathrm{N}-\mathrm{C}$ bond lengths and its binding energy (BE) strength of the vdW form of these complexes are almost invariant except for $\mathrm{C}_{14}$-pip which has the smallest BE. On the other hand, the bond distance of the DB complexes increases with increasing cyclo[n]carbon ring size, and their corresponding BEs decrease. The threshold barrier for the $\mathrm{vdW} \rightarrow \mathrm{DB}$ conversion is predicted to be lower with decreasing ring size and high exergonicity of the reaction showing Hammond's postulate type correlation. ${ }^{24}$ This trend in the $\Delta E^{\ddagger}$ and $\Delta E_{\mathrm{r}}$ is due to the fact that in going from $\mathrm{C}_{14}$ to $\mathrm{C}_{22}$, what Hobza termed the "strain energy", i.e., the energy required for ring deformation during complexation, increases, thereby explaining the higher $\Delta E^{\ddagger}$ and destabilizing $\Delta E_{\mathrm{r}}{ }^{23}$ Notably, we 
have also studied smaller complexes formed by $\mathrm{C}_{10}$ and $\mathrm{C}_{12}$; however, it exhibits a barrierless flat potential yielding a single minimum energy configuration with an $\mathrm{N}-\mathrm{C}$ bond distance of 1.496 and $1.487 \AA$ and $\mathrm{BE}$ of -39.4 and $-37.5 \mathrm{kcal} \mathrm{mol}^{-1}$ for the former and latter, respectively.

Table 2. N-C bond distance for van der Waals (vdW), dative bond (DB), and transition state (TS) structures in $\AA$, and their respective binding energies (BE) along with threshold energies (from LB to TS, $\left.\Delta E^{\ddagger}\right)$ and reaction energies $\left(\Delta E_{\mathrm{r}}\right)$ in $\mathrm{kcal} \mathrm{mol}^{-1}$, and transition state imaginary frequencies (v) in $\mathrm{cm}^{-1}$ and semiclassical $\left(k_{\mathrm{SC}}\right)$ and tunneling $\left(k_{\mathrm{T}}\right)$ rates in $\mathrm{s}^{-1}$ at $4 \mathrm{~K}$ for the studied $\mathrm{C}_{\mathrm{n}}-$ pip complexes.

\begin{tabular}{ccccccccccc}
\hline System & $\mathrm{vdW}$ & $\mathrm{DB}$ & $\mathrm{TS}$ & $\mathrm{BE}_{\mathrm{vdW}}$ & $\mathrm{BE}_{\mathrm{DB}}$ & $\Delta E^{\ddagger}$ & $\Delta E_{\mathrm{r}}$ & $v$ & $k_{\mathrm{SC}}$ & $k_{\mathrm{T}}$ \\
\hline $\mathrm{C}_{14}$-pip & 2.914 & 1.489 & 2.536 & -1.8 & -29.0 & 0.1 & -27.2 & $115 i$ & $6 \times 10^{5}$ & $7 \times 10^{9}$ \\
$\mathrm{C}_{16}$-pip & 2.934 & 1.501 & 2.380 & -2.3 & -23.5 & 1.2 & -21.2 & $150 i$ & $4 \times 10^{-56}$ & 100 \\
$\mathrm{C}_{18}$-pip & 3.016 & 1.496 & 2.307 & -2.1 & -19.7 & 2.2 & -17.7 & $164 i$ & $7 \times 10^{-110}$ & $9 \times 10^{-6}$ \\
$\mathrm{C}_{20}$-pip & 2.989 & 1.504 & 2.241 & -2.1 & -17.0 & 2.8 & -14.9 & $164 i$ & $2 \times 10^{-142}$ & $2 \times 10^{-7}$ \\
$\mathrm{C}_{22}$-pip & 3.016 & 1.505 & 2.207 & -2.0 & -14.6 & 3.5 & -12.6 & $207 i$ & $5 \times 10^{-182}$ & $9 \times 10^{-12}$ \\
\hline
\end{tabular}

Turning next to the QMT rates for the donor-acceptor complexes of these smaller analogs, our computations predict that the reactions are forbidden classically at $4 \mathrm{~K}$ except for $\mathrm{C}_{14}$, for which the reaction is almost barrierless, making the reaction feasible (See Table 2). However, the inclusion of tunneling correction in the overall rate constant accelerates the reaction by several orders of magnitude in all the studied cases. As shown in Table 2 , the $k_{\mathrm{T}}$ is extremely fast for the complexes with smaller $\mathrm{C}_{14}$ and $\mathrm{C}_{16}$ ring sizes, corresponding to a short half-live of $0.1 \mathrm{~ns}$ and 0.01 $\mathrm{s}$ at $4 \mathrm{~K}$ for the former and latter, suggesting that the vdW long-bond adduct is fleetingly stable. 
For larger $\mathrm{C}_{20}$ and $\mathrm{C}_{22}$ systems, the QMT rate is lower than for their smaller analogs (Table 2) with extremely more prolonged $t_{1 / 2}$ ranging from months to millennia at $4 \mathrm{~K}$, indicating that only the vdW adduct will be isolable in a standard experimental setup. However, it should be mentioned that as the temperature is raised to $50 \mathrm{~K}$, the role of thermally-activated tunneling enhances the overall reaction rate in all the above systems, making the vdW complex's existence fleeting (see Supporting Information for full rates table). For instance, the $k_{\mathrm{T}}$ for $\mathrm{C}_{20}$-pip and $\mathrm{C}_{22}$-pip results in a value of 0.7 and $5 \times 10^{-4} \mathrm{~s}^{-1}$ at $50 \mathrm{~K}$ with $t_{1 / 2}$ of $0.9 \mathrm{~s}$ and $\sim 20 \mathrm{~min}$. The slow QMT rates for the larger systems can be attributed to the higher threshold barrier and less exergonicity of the reaction, which can produce a wider barrier width.



Figure 3. Minimum-energy-path potential $\left(V_{\mathrm{mep}}\right)$ as a function of the reaction coordinate $(s)$ in mass-scaled coordinates for the studied $\mathrm{C}_{\mathrm{n}}-$ pip complexes.

Since an accurate estimation of the barrier width $(w)$ is difficult to determine - as real reactions do not exhibit a simple square or inverse parabolic PES, but instead run through a roughly gaussian-shaped minimum-energy-path $(\mathrm{MEP})^{25,59}$ - we therefore considered the potential energy profiles of the MEP $\left(V_{\text {mep }}\right)$ with respect to the mass-scaled reaction coordinate $(s)$ (see Fig. 3$)$ as a visual indicator of the influence of $w$ on the tunneling rate differences in $\mathrm{C}_{\mathrm{n}}$-pip systems. It can be 
seen clearly from Fig. 2 that with the increase in the size of the cyclocarbon ring, $w$ gets wider along with higher $\Delta E^{\ddagger}$, which nicely explains the reason for the decreasing QMT rates from $\mathrm{C}_{14-}$ pip to $\mathrm{C}_{22}$-pip.

\section{Conclusion}

In summary, our rate constant calculations including multidimensional tunneling correction reveal the overwhelming role of heavy atom QMT in the vdW $\rightarrow$ DB transformation of the $\mathrm{C}_{18}-$ pip complex at cryogenic temperatures where the classical over-the-barrier process is virtually non-existent. At the sublimation temperature of the argon matrix $(30 \mathrm{~K})$, the decay QMT rate is predicted to be extremely fast with a $t_{1 / 2}$ of only $38 \mathrm{~s}$, indicating the fleeting existence of vdW complex. The concave Arrhenius graph and the KIE analysis further sharpen the fingerprint of QMT in this reaction. Additionally, we explored the role of QMT in other $C_{n}-$ pip systems $(n=14$, 16, 20 and 22) and predicted a rapid QMT rate for the smaller analogs of $\mathrm{C}_{18}$, with very to extremely short $t_{1 / 2}$ ranging between seconds and nanoseconds at $4 \mathrm{~K}$. The present study shows that QMT can play a determining role in the covalent/dative bond formation of $\mathrm{C}_{18}$-pip. Considering the recent work on the enhanced stability of $\mathrm{C}_{60}$ with pip, it remains to be tested if tunneling can act a key player in the covalent/dative bond functionalization of fullerenes.

\section{ASSOCIATED CONTENT \\ Supporting Information}

The Supporting Information is available free of charge at https://pubs.acs.org/doi/10.1021/acs.xxx.yyyyyyy: 
Table of CVT and SCT rate constants as a function of temperature; Polyrate input and output details; all relevant geometries in XMol/Molden .XYZ format.

\section{AUTHOR INFORMATION}

\section{Corresponding Author}

Dr. Ashim Nandi, ashim.nandi@weizmann.ac.il

Prof. Dr. Jan M.L. Martin, gershom@weizmann.ac.il

\section{Funding Sources}

This research was supported in part by the Israel Science Foundation (grant 1969/20) and the Minerva Foundation (grant 2020/05).

\section{ACKNOWLEDGMENT}

A.N. acknowledges the Feinberg Graduate School for the Dean's Excellence Postdoctoral Fellowship.

\section{REFERENCES}

(1) Kaiser, K.; Scriven, L. M.; Schulz, F.; Gawel, P.; Gross, L.; Anderson, H. L. An SpHybridized Molecular Carbon Allotrope, Cyclo[18]Carbon. Science 2019, 365 (6459), 12991301. https://doi.org/10.1126/science.aay 1914 .

(2) Kroto, H. W.; Heath, J. R.; O’Brien, S. C.; Curl, R. F.; Smalley, R. E. C 60 : Buckminsterfullerene. Nature 1985,318 (6042), 162-163. https://doi.org/10.1038/318162a0.

(3) Iijima, S. Helical Microtubules of Graphitic Carbon. Nature 1991, 354 (6348), 56-58. https://doi.org/10.1038/354056a0.

(4) Novoselov, K. S.; Geim, A. K.; Morozov, S. V.; Jiang, D.; Zhang, Y.; Dubonos, S. V.; Grigorieva, I. V.; Firsov, A. A. Electric Field Effect in Atomically Thin Carbon Films. Science 2004. https://doi.org/10.1126/science.1102896.

(5) Baryshnikov, G. V.; Valiev, R. R.; Kuklin, A. V.; Sundholm, D.; Ågren, H. Cyclo[18]Carbon: Insight into Electronic Structure, Aromaticity, and Surface Coupling. J. Phys. Chem. Lett. 2019, 6701-6705. https://doi.org/10.1021/acs.jpclett.9b02815. 
(6) Nandi, A.; Solel, E.; Kozuch, S. Carbon Tunneling in the Automerization of Cyclo[18]Carbon. Chemistry - A European Journal 2020, 26 (3), 625-628. https://doi.org/10.1002/chem.201904929.

(7) Brémond, É.; Pérez-Jiménez, Á. J.; Adamo, C.; Sancho-García, J. C. Sp-Hybridized Carbon Allotrope Molecular Structures: An Ongoing Challenge for Density-Functional Approximations. J. Chem. Phys. 2019, 151 (21), 211104. https://doi.org/10.1063/1.5133639.

(8) Stasyuk, A. J.; Stasyuk, O. A.; Solà, M.; Voityuk, A. A. Cyclo[18]Carbon: The Smallest AllCarbon Electron Acceptor. Chem. Commun. 2020, 56 (3), 352-355. https://doi.org/10.1039/C9CC08399E.

(9) Hussain, S.; Chen, H.; Zhang, Z.; Zheng, H. Vibrational Spectra and Chemical Imaging of Cyclo[18]Carbon by Tip Enhanced Raman Spectroscopy. Chemical Communications 2020, 56 (15), 2336-2339. https://doi.org/10.1039/C9CC09130K.

(10) Lu, T.; Chen, Q. Ultrastrong Regulation Effect of the Electric Field on the All-Carboatomic Ring Cyclo[18]Carbon**. ChemPhysChem 2021, 22 (4), 386-395. https://doi.org/10.1002/cphc.202000903.

(11) Qin, B.; Zhang, Q.; Li, Y.; Yang, G.; Yu, H.; Peng, F. Mechanistic Insights into the Electrochemical Reduction of $\mathrm{CO} 2$ on Cyclo[18]Carbon Using Density Functional Theory Calculations. ChemElectroChem 2020, 7 (8), 1838-1842. https://doi.org/10.1002/celc.202000180.

(12) Zhang, L.; Li, H.; Feng, Y. P.; Shen, L. Diverse Transport Behaviors in Cyclo[18]CarbonBased Molecular Devices. The Journal of Physical Chemistry Letters 2020. https://doi.org/10.1021/acs.jpclett.0c00357.

(13) Jiang, Y.; Jun Mattioli, E.; Calvaresi, M.; Wang, Z. Theoretical Design of an Ultrafast Supramolecular Rotor Composed of Carbon Nano-Rings. Chemical Communications 2020, 56 (79), 11835-11838. https://doi.org/10.1039/D0CC04806B.

(14) Fang, S.; Hu, Y. H. Cyclo[18]Carbon as an Ultra-Elastic Molecular O-Ring with Unique Mechanical Properties. Carbon 2021, 171, 96-103. https://doi.org/10.1016/j.carbon.2020.08.082.

(15) Liu, Z.; Lu, T.; Chen, Q. Intermolecular Interaction Characteristics of the All-Carboatomic Ring, Cyclo[18]Carbon: Focusing on Molecular Adsorption and Stacking. Carbon 2021, 171, 514-523. https://doi.org/10.1016/j.carbon.2020.09.048.

(16) Dai, C.; Chen, D.; Zhu, J. Achieving Adaptive Aromaticity in Cyclo[10]Carbon by Screening Cyclo[n]Carbon (N=8-24). Chemistry - An Asian Journal 2020, 15 (14), 2187-2191. https://doi.org/10.1002/asia.202000528.

(17) D. Charistos, N.; Muñoz-Castro, A. Induced Magnetic Field in Sp-Hybridized Carbon Rings: Analysis of Double Aromaticity and Antiaromaticity in Cyclo[2 N ]Carbon Allotropes. Physical Chemistry Chemical Physics 2020, 22 (17), 9240-9249. https://doi.org/10.1039/D0CP01252A.

(18) Liu, Z.; Lu, T.; Chen, Q. An Sp-Hybridized All-Carboatomic Ring, Cyclo[18]Carbon: Bonding Character, Electron Delocalization, and Aromaticity. Carbon 2020, 165, 468-475. https://doi.org/10.1016/j.carbon.2020.04.099.

(19) Lamanec, M.; Lo, R.; Nachtigallová, D.; Bakandritsos, A.; Mohammadi, E.; Dračínský, M.; Zbořil, R.; Hobza, P.; Wang, W. The Existence of a $\mathrm{N} \rightarrow \mathrm{C}$ Dative Bond in the C60-Piperidine Complex. Angewandte Chemie 2021, 133 (4), 1970-1978. https://doi.org/10.1002/ange.202012851. 
(20) Beck, M. T.; Mándi, G. Solubility of C 60. Fullerene Science and Technology 1997, 5 (2), 291-310. https://doi.org/10.1080/15363839708011993.

(21) Talukdar, S.; Pradhan, P.; Banerji, A. Electron Donor-Acceptor Interactions of C60 with nAnd $\pi$-Donors: A Rational Approach Towards Its Solubility. Fullerene Science and Technology 1997, 5 (3), 547-557. https://doi.org/10.1080/15363839708015907.

(22) Solís-Fernández, P.; Okada, S.; Sato, T.; Tsuji, M.; Ago, H. Gate-Tunable Dirac Point of Molecular Doped Graphene. ACS Nano 2016, $10 \quad$ (2), 2930-2939. https://doi.org/10.1021/acsnano.6b00064.

(23) Lo, R.; Manna, D.; Hobza, P. Cyclo[n]Carbons Form Strong N $\rightarrow$ C Dative/Covalent Bonds with Piperidine. J. Phys. Chem. A 2021, 125 (14), 2923-2931. https://doi.org/10.1021/acs.jpca.1c01161.

(24) Anslyn, E. V.; Dougherty, D. A. Modern Physical Organic Chemistry; University Science, Sausalito, CA, 2005.

(25) Castro, C.; Karney, W. L. Heavy-Atom Tunneling in Organic Reactions. Angew. Chem. Int. Ed. 2020, 59 (22), 8355-8366. https://doi.org/10.1002/anie.201914943.

(26) Meisner, J.; Kästner, J. Atom Tunneling in Chemistry. Angew. Chem. Int. Ed. 2016, 55 (18), 5400-5413. https://doi.org/10.1002/anie.201511028.

(27) Nandi, A.; Kozuch, S.; Kästner, J. Comment on "Computational Evidence for Sulfur Atom Tunneling in the Ring Flipping Reaction of S4N4." Chem. Phys. Lett. 2020, 754, 137678. https://doi.org/10.1016/j.cplett.2020.137678.

(28) Borden, W. T. Reactions That Involve Tunneling by Carbon and the Role That Calculations Have Played in Their Study. WIREs Computational Molecular Science 2016, 6 (1), 20-46. https://doi.org/10.1002/wcms.1235.

(29) Karmakar, S.; Datta, A. Heavy-Atom Tunneling in Organic Transformations. J Chem Sci 2020, 132 (1), 127. https://doi.org/10.1007/s12039-020-01809-x.

(30) Bell, R. P. The Tunneling Effect in Chemistry; Chapman and Hall, London, 1980.

(31) Schreiner, P. R. Quantum Mechanical Tunneling Is Essential to Understanding Chemical Reactivity. Trends Chem. 2020, 2 (11), 980-989. https://doi.org/10.1016/j.trechm.2020.08.006.

(32) Greer, E. M.; Cosgriff, C. V.; Doubleday, C. Computational Evidence for Heavy-Atom Tunneling in the Bergman Cyclization of a 10-Membered-Ring Enediyne. J. Am. Chem. Soc. 2013, 135 (28), 10194-10197. https://doi.org/10.1021/ja402445a.

(33) Schleif, T.; Mieres-Perez, J.; Henkel, S.; Ertelt, M.; Borden, W. T.; Sander, W. The Cope Rearrangement of 1,5-Dimethylsemibullvalene-2(4)-D1: Experimental Evidence for HeavyAtom Tunneling. Angewandte Chemie International Edition 2017, 56 (36), 10746-10749. https://doi.org/10.1002/anie.201704787.

(34) Zhang, X.; Hrovat, D. A.; Borden, W. T. Calculations Predict That Carbon Tunneling Allows the Degenerate Cope Rearrangement of Semibullvalene to Occur Rapidly at Cryogenic Temperatures. Org. Lett. 2010, 12 (12), 2798-2801. https://doi.org/10.1021/ol100879t.

(35) Kozuch, S. Heavy Atom Tunneling in the Automerization of Pentalene and Other Antiaromatic Systems. RSC Advances 2014, 4 (41), 21650-21656. https://doi.org/10.1039/C4RA02191F.

(36) Nandi, A.; Sucher, A.; Kozuch, S. Ping-Pong Tunneling Reactions: Can Fluoride Jump at Absolute Zero? Chemistry - A European Journal 2018, 24 (61), 16348-16355. https://doi.org/10.1002/chem.201802782. 
(37) Sedgi, I.; Kozuch, S. Heavy Atom Tunnelling on XeF6 Pseudorotation. Phys. Chem. Chem. Phys. 2020, 22 (31), 17725-17730. https://doi.org/10.1039/D0CP03205K.

(38) Nandi, A.; Sucher, A.; Tyomkin, A.; Kozuch, S. Ping-Pong Tunneling Reactions, Part 2: Boron and Carbon Bell-Clapper Rearrangement. Pure Appl. Chem. 2020, 92 (1), 39-47. https://doi.org/10.1515/pac-2019-0401.

(39) Schreiner, P. R. Tunneling Control of Chemical Reactions: The Third Reactivity Paradigm. J. Am. Chem. Soc. 2017, 139 (43), 15276-15283. https://doi.org/10.1021/jacs.7b06035.

(40) Gerbig Dennis; Schreiner Peter R. Tunneling in the Reactions of Carbenes and Oxacarbenes. Contemporary Carbene Chemistry 2013. https://doi.org/10.1002/9781118730379.ch7.

(41) Nunes, C. M.; Eckhardt, A. K.; Reva, I.; Fausto, R.; Schreiner, P. R. Competitive Nitrogen versus Carbon Tunneling. J. Am. Chem. Soc. 2019, 141 (36), 14340-14348. https://doi.org/10.1021/jacs.9b06869.

(42) Nandi, A.; Tarannam, N.; Rodrigues Silva, D.; Fonseca Guerra, C.; Hamlin, T. A.; Kozuch, S. Boron Tunneling in the "Weak" Bond-Stretch Isomerization of N-B Lewis Adducts. ChemPhysChem 2021, 22 (18), 1857-1862. https://doi.org/10.1002/cphc.202100505.

(43) Zhao, Y.; Truhlar, D. G. The M06 Suite of Density Functionals for Main Group Thermochemistry, Thermochemical Kinetics, Noncovalent Interactions, Excited States, and Transition Elements: Two New Functionals and Systematic Testing of Four M06-Class Functionals and 12 Other Functionals. Theor. Chem. Acc. 2008, 120 (1), 215-241. https://doi.org/10.1007/s00214-007-0310-X.

(44) M. J. Frisch, G. W. Trucks, H. B. Schlegel, G. E. Scuseria, M. A. Robb, J. R. Cheeseman, G. Scalmani, V. Barone, G. A. Petersson, H. Nakatsuji, X. Li, M. Caricato, A. V. Marenich, J. Bloino, B. G. Janesko, R. Gomperts, B. Mennucci, H. P. Hratchian, J. V. Ortiz, A. F. Izmaylov, J. L. Sonnenberg, D. Williams-Young, F. Ding, F. Lippa-rini, F. Egidi, J. Goings, B. Peng, A. Petrone, T. Hender-son, D. Ranasinghe, V. G. Zakrzewski, J. Gao, N. Rega, G. Zheng, W. Liang, M. Hada, M. Ehara, K. Toyota, R. Fukuda, J. Hasegawa, M. Ishida, T. Nakajima, Y. Honda, O. Kitao, H. Nakai, T. Vreven, K. Throssell, J. A. Mont-gomery, Jr., J. E. Peralta, F. Ogliaro, M. J. Bearpark, J. J. Heyd, E. N. Brothers, K. N. Kudin, V. N. Staroverov, T. A. Keith, R. Kobayashi, J. Normand, K. Raghavachari, A. P. Rendell, J. C. Burant, S. S. Iyengar, J. Tomasi, M. Cossi, J. M. Millam, M. Klene, C. Adamo, R. Cammi, J. W. Ochterski, R. L. Martin, K. Morokuma, O. Farkas, J. B. Foresman and D. J. Fox, Gaussian 16, Revision A.03, Gaussian, Inc., Wallingford, CT 2016.

(45) Martin, J. M. L.; Bauschlicher, C. W.; Ricca, A. On the Integration Accuracy in Molecular Density Functional Theory Calculations Using Gaussian Basis Sets. Computer Physics Communications 2001, 133 (2), 189-201. https://doi.org/10.1016/S0010-4655(00)00174-0.

(46) D G Truhlar; Garrett, and B. C. Variational Transition State Theory. Annu. Rev. Phys. Chem. 1984, 35 (1), 159-189. https://doi.org/10.1146/annurev.pc.35.100184.001111.

(47) Fernandez-Ramos, A.; Ellingson, B. A.; Garrett, B. C.; Truhlar, D. G. Variational Transition State Theory with Multidimensional Tunneling. In Reviews in Computational Chemistry; Wiley-Blackwell, 2007; pp 125-232. https://doi.org/10.1002/9780470116449.ch3.

(48) Skodje, R. T.; Truhlar, D. G.; Garrett, B. C. Vibrationally Adiabatic Models for Reactive Tunneling. J. Chem. Phys. 1982, 77 (12), 5955-5976. https://doi.org/10.1063/1.443866.

(49) J. Zheng, S. Zhang, B. J. Lynch, J. C. Corchado, Y.-Y. Chuang, P. L. Fast, W.-P. Hu, Y. -P. Liu, G. C. Lynch, K. A. Nguyen, C. F. Jackels, A. F. Ramos, B. A. Ellingson, V. S. Melissas, J. Villà, I. Rossi, E. L. Coitiño, J. Pu, T. V. Albu,. POLYRATE, version 2016-2A, University of Minnesota, Minneapolis, MN, 2016. 
(50) J. Zheng, S. Zhang, J. C. Corchado, Y. Y. Chuang, E. L. Coitino, B. A. Ellingson, Truhlar, D. G. GAUSSRATE, version 2016, University of Minnesota, Minneapolis, MN, 2016.

(51) Page, M.; McIver, J. W. On Evaluating the Reaction Path Hamiltonian. J. Chem. Phys. 1988, 88 (2), 922-935. https://doi.org/10.1063/1.454172.

(52) Lauderdale, J. G.; Truhlar, D. G. Diffusion of Hydrogen, Deuterium, and Tritium on the (100) Plane of Copper: Reaction-Path Formulation, Variational Transition State Theory, and Tunneling Calculations. Surf. Sci. 1985, 164 (2), 558-588. https://doi.org/10.1016/00396028(85)90766-6.

(53) J. Tomasi, B. Mennucci and R. Cammi. Chem. Rev 2005, 105, 2999-3093.

(54) Amlani, H.; Frenklah, A.; Kozuch, S. Chapter 2: Tunnelling Instability in Molecular Systems. An Exercise in Computational Chemistry Prediction Power. In Tunnelling in Molecules; 2020; pp 61-87. https://doi.org/10.1039/9781839160370-00061.

(55) Greer, E. M.; Kwon, K.; Greer, A.; Doubleday, C. Thermally Activated Tunneling in Organic Reactions. Tetrahedron 2016, 72 (47), 7357-7373. https://doi.org/10.1016/j.tet.2016.09.029.

(56) Kozuch, S. The Reactivity Game: Theoretical Predictions for Heavy Atom Tunneling in Adamantyl and Related Carbenes. Phys. Chem. Chem. Phys. 2014, 16 (17), 7718-7727. https://doi.org/10.1039/C4CP00115J.

(57) Lee, T. J.; Taylor, P. R. A Diagnostic for Determining the Quality of Single-Reference Electron Correlation Methods. Int. J. Quantum Chem. 1989, 36 (S23), 199-207. https://doi.org/10.1002/qua.560360824.

(58) Fogueri, U. R.; Kozuch, S.; Karton, A.; Martin, J. M. L. A Simple DFT-Based Diagnostic for Nondynamical Correlation. Theor. Chem. Acc. 2013, 132 (1), 1291. https://doi.org/10.1007/s00214-012-1291-y.

(59) Nandi, A.; Alassad, Z.; Milo, A.; Kozuch, S. Quantum Tunneling on Carbene Organocatalysis: Breslow Intermediate Formation via Water-Bridges. ACS Catal. 2021, 11 (24), 14836-14841. https://doi.org/10.1021/acscatal.1c04475. 